\section{AN OPERATION FOR HYPOSPADIAS.}

BY ARTHUR EDMUNDS, M.B., M.S., B.Sc. LOND., F.R.C.S. ENG.,

SURGEON TO THE GREAT NOR'LHERN CENTRAL HOSPITAL; ASSISTAN' SURGEON TO KING'S COLLEGE HOSPITAL.

HyPOSPADIAS is a condition which varies in degree from a mere elongation of the external meatus to a complete cleft of the urethra as far back as the perineum. In the type of case for which an operation is most commonly performed, the opening of the urethra is situated just in front of the scrotum. The penis is strongly curved, and when an attempt is made to straighten the organ, the imperfectly urethra and in obtaining a permanent straightening of the penis.

The superficial tissues of the penis are extremely lax, and hence it is possible to perform a plastic operation, which would be impossible in dealing with more rigid tissues; but at the same time union is hindered by the constant passage of urine, and hence it is necessary to provide for the adequate nutrition of the flaps by every possible means. In the operation about to be described a new urethra is formed out of the prepuce, which in hypospadias is represented by a large hooded flap of tissue on the dorsum of the penis. The prepuce is chiefly vascularised by one or more vessels which enter it on the dorsum of the penis near the middle line. The operation is performed in three stages.

Stage 1.-The first step of the operation consists in
FIG. 1.

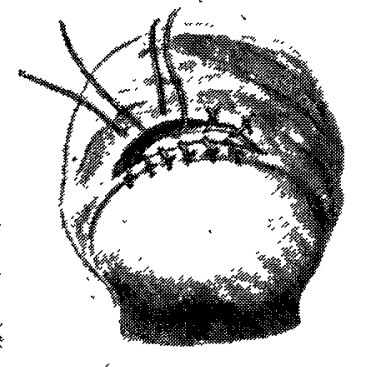

Formation of the buttonhole in the prepuce, showing the method of pessing sutures, two of which are left untied.
FIG. 2.

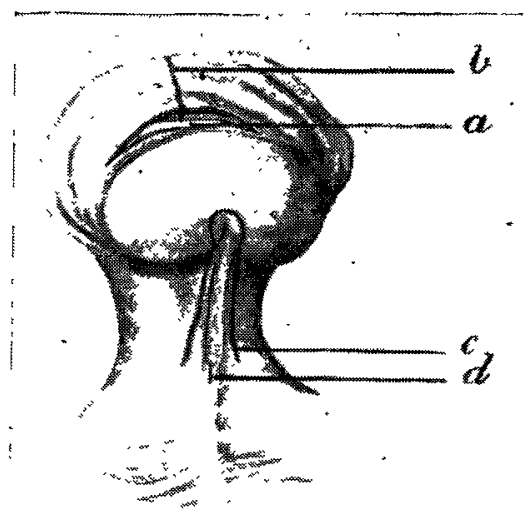

The incisions for the second stage of the operation. $a$, The buttonhole. $b$, Incision through the prepuee. $c$, Incision around the urethral groove. $d$, Incision through flap of mucous membrane at opening of urethra.
FIG. 3.

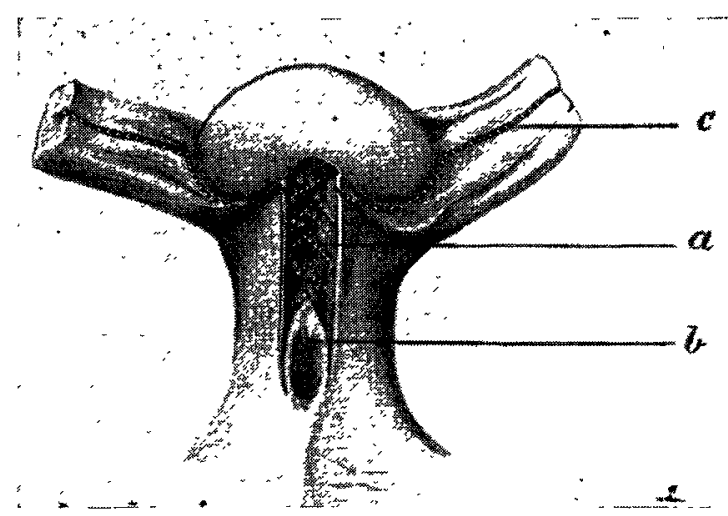

The urethra detached. $a$. Incisions for the detachment of the urethra. $b$, The shrunken urethral groove. $c$, Incision, on the Ieft side, around the glans and along the preputial flap. developed urethra stands out as a definite cord. The disabilities which this deformity entails are, that during micturition the stream of urine is projected downwards and back. wards and soils the patient's clothes; that the curvature of the organ and the position of the urethral orifice render coitus difficult, and in addition, that the presence of a misshapen penis is apt to have a harmful mental effect on the

FIG. 4.

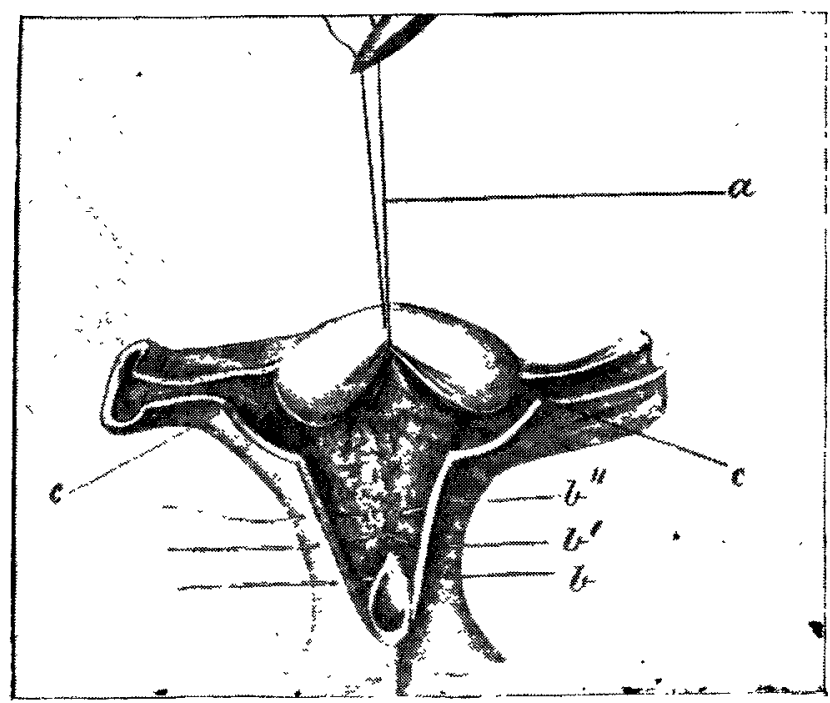

Detachment of the flaps. $a$, Guide stitch through the glans. $b$, First stitch. $b^{\prime}$, Second stitch. $b^{\prime \prime}$, Third stitch. $c c$, Points on the flaps which will form the most anberior part of the roof of the urethra.

patient, leading him to fancy himself fundamentally different from the rest of mankind.

The objects of the operation must therefore be to straighten the organ and to provide the urethra with an outlet at the end of the penis. The formation of a floor to the urethra in the body of the penis can be accomplished fairly easily, but diffieulties are encountered in attempting to form the glandular making an incision (Fig. 1) through the whole thickness of the prepuce, so as to form a sort of buttonhole, which is then stitched round in much the same way as a tailor finishes off a buttonhole in a coat. The object of this incision is to divide the dorsal vessels of the prepuce, and so lead to the formation of a number of smaller vessels, which will vascularise the prepuce from the sides. The wound thus made should be allowed to heal and the resulting cicatrix to soften before the second stage of the operation is performed - that is to say, about three months should be allowed to elapse.

Stage 2.-An incision is now made through the prepuce from the middle of the buttonhole to the free border, so as to form two irregular flaps (Fig. $3 c)$. Two incisions are now made, one along each side of the urethral groove ; these are joined in front by an incision in the glans around the point where the meatus would normally be situated. the whole forming a long inverted U-shaped incision around the urethral groove (Fig.
FIG. 5.

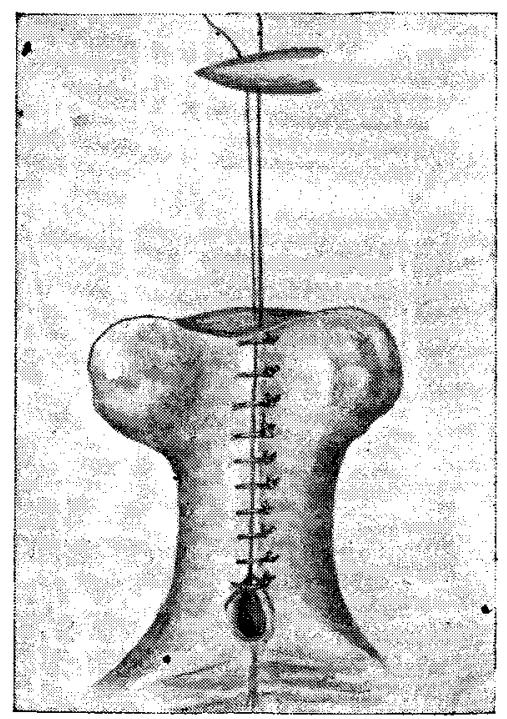

Completion of the second stage. Ventral aspect of the penis.
$2, c)$. The opening of the urethra is sometimes constricted, or bounded by a thin fold of mucous membrane, which should be divided by a short incision (Fig. $2, d$ ). The incision around the urethral groove is now deepened so as to dissect up this structure completely; this is best done by a series of short cuts made in the direction shown in Fig. 3. a. When this has been carried out 
the condition shown in Fig. 3 will be produced-that is to say, the penis will be straight, and there will be a broad raw surface on the under aspect, at the scrotal end of which the urethra opens. The detached urethral groove does not remain as an elongated flap, but contracts into a surprisingly small mass (Fig. $3, b$ ). An incision is then made on each side from the anterior portion of the raw area around the corona glandis, and about a millimetre away from this structure until the middle of the preputial flap is reached, when the direction of the incision is altered and it is carried along the long axis of the flap up to the free edge which was made by dividing the buttonhole (Fig 3,o). This incision should extend just through the skin, and it will then be possible to open out the lax tissues so as to produce the rubber catheter is passed into the urethra and stitched to the middle line of the glans (Fig. 7,a); this steadies the organ and facilitates the rest of the operation. An incision is made on either side of the urethral channel, extending into the connective tissue, which is then sutured around the bougie by a row of stitches (Fig. 7, b) ; the skin is closed over this by another row of stitches (Fig. 7. c). At the anterior part care should be taken to make the incision run slightly outwards, so as to make the floor of the urethra slightly longer than the roof ; this allows for shrinkage.

The operation is now finished, and the appearance of the penis is that of an organ which has been circumcised, leaving a rather redundant mass of tissue below the frenum (Fig. 8). If everything heals satisfactorily the operation is completed,

FIG. 7.

FIG. 6.

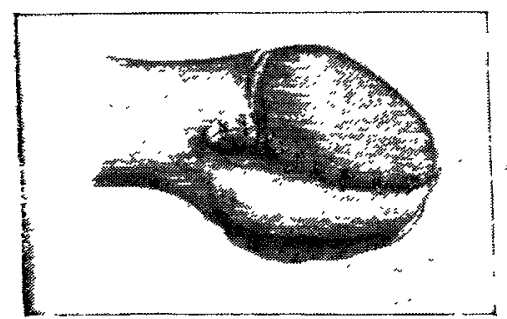

Lateral view of Fig. 5 .

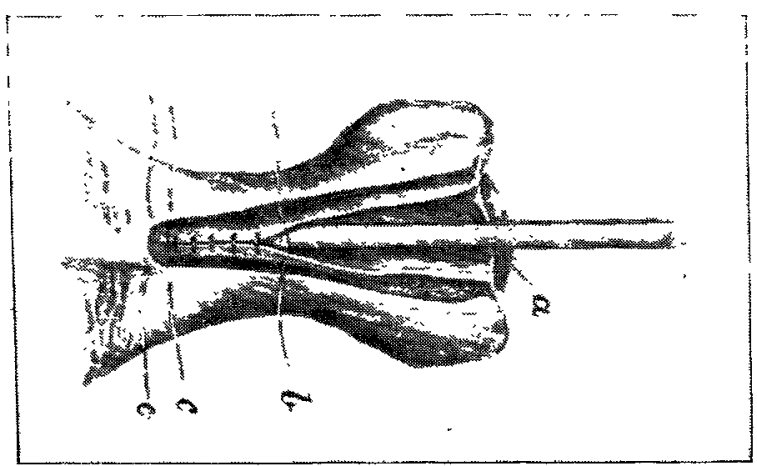

The third stage of the operation. $a$, Catheter stitched into meatus. $b$, Deep sutures (catgut). $c$, Superficial skin
FIG. 8.

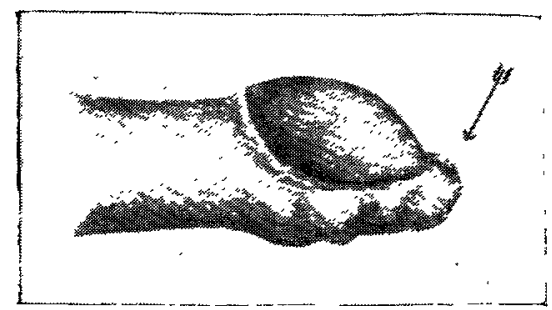

The completed operation. The arrow points to the urethral orifice conditions shown in Fig. 4 ; this should be done with as little actual division of tissue as possible.

The next proceeding. consists in passing stitches so as to slide in skin from the preputial flaps and thus cover the whole of this raw area with epithelium, part of which will ultimately line the new urethra. It is well to pass a stitch through the glans so as to hold the organ straight before passing the sutures. The first of these (Fig. $4, b$ ) is placed so as to pick up the point of the shrunken uretinra; the next (Fig. 4, $b^{\prime}$ ) passes through the skin on one side, takes a hold on the fibrous tissue of the raw area, and finally emerges through the skin on the opposite side; the remaining sutures are passed similarly, the flaps being brought as far towards the orifice of the urethra as can be done withot except that there will usually be a few rather unsightly tags of skin at the side of the glans, which can be clipped away if desired; but this should never be done until sound union has been obtained, as these tags will prove extremely valnable if it should be found necessary to perform any further plastic operation for closing a fistula. Fistulæ most commonly occur either at the junction of the body of the penis with the glans or just in front of the scrotum, and they are best closed in the manner shown in Figs. 10, 11, and 12. An incision is made round the fistula in the manner shown in Fig. 10, in which it will be noticed that the incision is not symmetrically placed in regard to the fistulous opening, but is well to one side, so that a, flap is raised, which can be turned over the opening and sutured in position (Fig. 11)
FIG. 9.

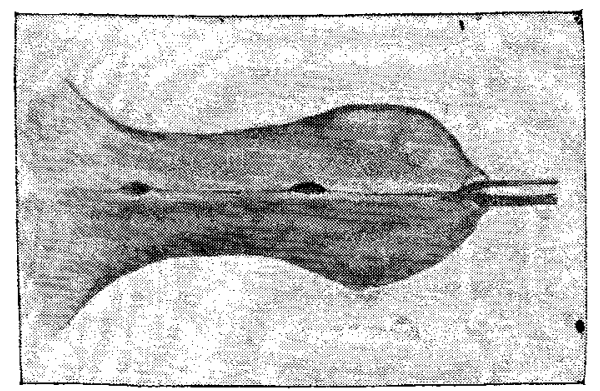

To show the most common position in which fistulæ form.

puckering. It will usually be found that the points $c e$ (Fig. 4) will be sutured to the raw surface, just below the stitch which has been passed through the glans. The appearance when all these stitches have been passed is seen in Fig. 5. On the lateral aspect of the glans there is still a line along which the epithelium is not united, and this should now be done by a row of sutures (Fig. 6). This completes the second stage of the operation, and when the wounds have healed it will be found that the penis is perfectly straight and considerably broader, especially in the region of the glans.

Stage 3.-At least another three months should be left before commencing this stage of the operation, which is carried out almost exactly as in Duplay's operation. A soft
FIG. 11.

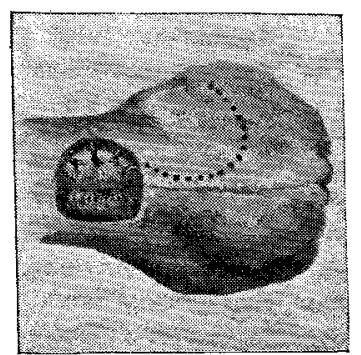

FIG. 12.

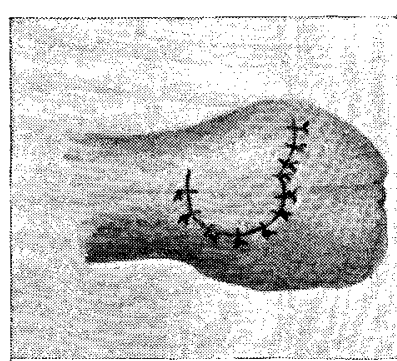

Fras. 10, 11, 12.-Method of closing a fistula.

The free edge of this flap should be distinctly longer than the fistulous opening, so as to allow for shrinkage and for the insertion of sutures. When the flap has been fastened in position a raw surface is left, which is closed by sliding in a curved flap, as marked out in Fig. 11, the whole being sutured into position. This flap will usually be composed of the redundant tissue on one side of the glans, which will now present the appearance shown in Fig. 12. In the case of fistulæ at the root of the scrotum double flaps are cut as before, but the second flap is derived from scrotal tissue.

As regards suture material I have always employed fine chromicised catgut, except in the case of the final skin sutures, for which I have used horsehair. The parts are extremely delicate and friable, and it is therefore necessary to use fine 
needles, preferably ordinary sewing needles or curved intestinal needles. Accuracy in suturing is, of course, indispensable, but it is equally important to get a good hold on the skin flap, so that the stitches will not cut out when they are moistened with urine. As regards dressings, strips of moist boric lint wrapped round the organ and changed as often as they are soiled with urine are probably the most satisfactory, and are certainly the most comfortable for the patient. I have not found any particular advantage in keeping a catheter tied in to prevent the passage of urine over the wound. An instrument tends to set up urethritis and to keep a certain amount of tension on the stitches; furthermore, it does not always succeed in keeping the wound dry, as urine is apt to leak by the side of it.

The operation can be performed at any time after the age of 3 , but it is certainly easier when the boy is older, as the parts are larger and simpler to handle. In the cases which I have completed the results are extremely satisfactory. The urine is passed freely and projected forwards in a satisfactory stream, the straightness of the organ being all that could be desired. The new lining of the urethra, as seen in those cases in which a fistulous opening formed, seems to have taken on the character of mucous membrane. I have now operated on five cases; two are completely healed, one has a fistulous opening which remains to be closed, and in two the third stage has not yet been attempted; in all, however, the straightness of the organ is entirely satisfactory.

Queen Anne-street, W.

\section{ON A CASE OF ADDISON'S DISEASE IN A BOY AGED TEN YEARS. ${ }^{1}$}

\section{BY FREDERICK LANGMEAD, M.D. LOND.,} M.R.C.P. LOND.,

ASSISTANT PHYSICIAT TO THE ROYAT FREE HOSPITAL; THE HOSPITAY FOR SICK CHILDREN, GREAT ORMOND STREE' ; AND THE SEAMEN'S HOSPITAL SOCIETY (DREADYOUGHT), GREEYWICH.

DEFINITE cases of Addison's disease in children are without doubt very rare. In the annals of the Hospital for Sick Children, Great Ormond-street, no record appears of a post-mortem examination on a patient who had been the subject of this disease, nor is there any clinical record of a case in which the symptoms were convincing enough to make the diagnosis more than questionable. Comby has collected 21 cases in children, and Dezirot 48 cases in children under 16 . Of these, only 6 were under 10, the majority being in the eleventh and fifteenth years of life. Osler records an example of the disease occurring congenitally. Monti was able to find records of 11 cases in children from 3 to 14 years old. The disease in children would appear to produce symptoms very similar to those occurring in adults. Although protracted cases are described, a greater proportion run a very acute course in children. Apparently the pigmentation is nsually less obvious than in the majority of adult cases, although, as is well known, a few of the latter show little or no darkening of the skin or mucous membrane. Death from convulsions and coma would appear also to be relatively more frequent in children and death from asthenia relatively less. According to Gerhardt convulsions are seen in twofifths of the cases in children. Netter describes a case in a child, aged 4 years, who was suddenly taken ill with vomiting, diarrhœea, and depression, and died in three days.

The following case has many interesting features. The patient, a boy aged 10, was admitted to the Royal Free Hospital on Sept. 17th, 1912, at 6 A. Ir., and died four hours after admission. On the evening before, vomiting had started abruptly, accompanied by restlessness, and followed by loss of consciousness. On admission he was seen to be unconscious, the elbows, wrists, and knees being rigidly flexed, and the fingers extended. The temperature was subnormal and the pulse rapid and weak. The abdomen was retracted. The knee-jerks were present, and the plantar response was flexor. Frequent convulsive seizures occurred until death.

The case gave rise to much conjecture as to its nature,

1 The specimen was shown at the Medical Society of London on Jan. 27 th, 1913.
J and remained undiagnosed until the post-mortem examination. A possible explanation appeared to be meningitis, but the rapidity of its course was against this view. Moreover, the optic dises were normal, and cerebro-spinal fluid withdrawn by lumbar puncture showed no abnormality beyond absence of reducing power. The possibility of uræmia was excluded by the fact that a catheter specimen of urine was normal. Acetone was not present, an observation which excluded "cyclical vomiting" from the diagnosis. The pigmentation amounted to no more than a slight diffuse brownness of the skin, including that of the abdomen, and would not give rise to comment in a child of the poor at the end of the summer. Later it was learnt by careful questioning of the mother that the pigmentation had been noticed to have gradually deepened for about 12 months, and that the boy had been subject to attacks of diarrhoea for "several months," and had been slowly getting weaker. The boy's father had died from phthisis ten years before, and two aunts and one uncle of his mother also clied from the same disease.

Necropsy. - The body was that of a somewhat wasted boy, the skin showing slight general pigmentation without maculæ.

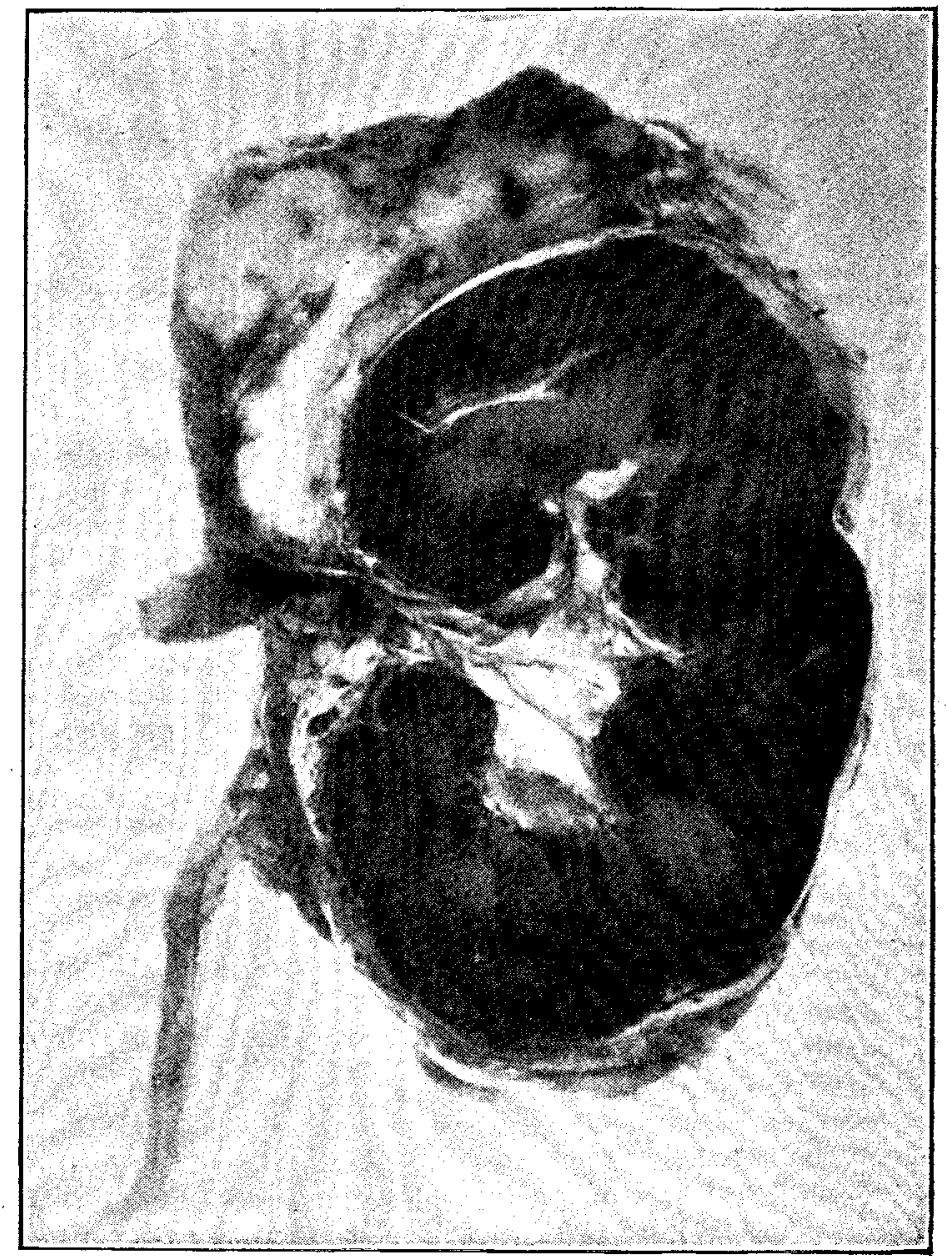

From a photograph of one kidney and suprarenal gland showing the advancen statre of the tibro-caseous change. The othe was similarly affectet. (Photograph by Miss Katherine Wortham.

The suprarenal glands were fibro-caseous, almost throughout, merely a narrow maroin of healthy gland tissue being discernible, and this only in a few areas (see figure). The fibrosis had spread into the adjoining posterior abdominal parietes, and must have involved the sympathetic ganglia, though these were not recognised. Caseous glands were found in the portal fissure and two caseous foci in the lowest lobes of the lungs, near the roots, probably representing glands also. Microscopically the tuberculous nature of the process was apparent. Many large giant cell systems and tubercle bacilli were seen. There was very considerable overgrowth of the lymphatic structures. The thymus, tonsils, lymphatic patches of the tongue and intestine, and the mesenteric glands were all hpperplastic, as in status lymphaticus. The spleen was considerably enlarged, reaching well down into the abdomen almost to the umbilicus, and weighing $5 \mathrm{oz}$ The pancreas, thyroid, thymus, spleen, and pituitary body 